\title{
Interest in the early extraction of temporary maxillary canines to prevent the inclusion of permanent canines and determination of the Canine Congestion Index ${ }^{\circledR}$. Radiological retrospective study
}

\author{
Ch. Fouquet, C. Philip-Alliez, M. Le Gall \\ ODF Functional Unit, Timone Hospital, Odontology Department, 264 rue Saint-Pierre, \\ 13385 Marseille, France
}

\begin{abstract}
SUMMARY
This study is designed to demonstrate the interest in extracting temporary maxillary canines to facilitate the establishment of permanent canines in ectopic positions. On the basis of the results of our experience with 104 maxillary canines in ectopic positions in 58 patients aged 8-15 years, we recommend using temporary canines and radiological follow-up for a period of 18 months (interval of 6 months) with orthopantomograms to monitor the development of permanent canines lanteroposterior position, angle, and vertical position). We found an improvement in the positioning of the permanent canines through the extraction of deciduous canines in $87 \%$ cases. On the basis of these results, we devise an interception scored protocol of ectopic canines by extraction of deciduous canines with other inclusion criteria, which is defined as the Canine Crowding Index ${ }^{\circledR}$. There is significant interest in avoiding included canines using an orthopantomogram, which is important for both the patient and the practitioner; this results in a decrease in treatment time, orthosurgical traction failures, and root resorption of the adjacent teeth.
\end{abstract}

\section{KEYWORDS}

Ectopic maxillary canine, temporary maxillary canine extraction, orthodontic treatment, radiographic assessment

\section{INTRODUCTION}

The treatment of included teeth has long been synonymous with extraction. However, the introduction of surgical removal and orthodontic traction instead of a more conservative approach by 1975 resulted in a more optimized treatment of included

Address for correspondence:

Michel Le Gall

264 rue Saint-Pierre - 13385 Marseille - France

E-mail: msjlegall@aol.com

Article received: 20-04-2017. Accepted for publication: 01-06-2017.

This is an Open Access article distributed under the terms of the Creative Commons Attribution License (http://creativecommons.org/licenses/by/4.0), which permits unrestricted use, distribution, and reproduction in any medium, provided the original work is properly cited. 
maxillary canines ${ }^{4}$. Orthodontic treatments, according to our current philosophy, can only be used on young adult teeth. In fact, although genetic origin of malocclusions predominates, a better knowledge of craniofacial growth nowadays leads us to start orthodontic treatments earlier, especially in mixed dentition. The terms preventive and interceptive treatment are today essential in our practice because they allow us in many cases to avoid major treatments like the orthosurgical treatment of included canines.

A prophylactic approach to eruptive problems should be favored by recognizing the precursory clinical signs, such as an incompressible and painless maxillary arch.

Pathological images and ectopic pathways should be diagnosed early with radiological assessments.

\section{MATERIALS AND METHODS}

\section{Study outline}

This is a retrospective analytical study carried out in the Dento-facial Orthopedics Department of the $\mathrm{CHU}$ de $\mathrm{La}$ Timone des docteurs Le Gall et PhilipAlliez (Le Timone University Hospital by Doctors Le Gall and Philip-Alliez) in Marseille as part of a dentofacial orthopedics thesis.

The patients consulted at the office of Dr. Gall-Bachet-Dameron-Chevalier in Aubagne. The number of patients included was 58. This study allowed us to study, using panoramic images, the variation of the position of 104 permanent canines in ectopic position after the extraction of temporary canines.
As soon as an anomaly has been detected, a suitable therapeutic protocol must be implemented, such as close clinical and radiological monitoring, extraction of temporary canines, removal of anatomical obstacles, and increase of the arch perimeter (increased transverse and/or anteroposterior dimension) ${ }^{1}$. The objective of this study was to evaluate the radiological placement of maxillary permanent canines in the ectopic position after temporary canine extractions in the absence of any other orthodontic treatment.

Our results reveal that the extraction of temporary canines is an optimal prophylactic approach to avoid the impaction of canines that present an ectopic path.

An orthopantomogram is performed just before the T0 extractions and then at 6-month intervals.

\section{Study population}

\section{Inclusion criteria}

The inclusion criteria for this study are:

- consultant subject in the office of Dr. Le Gall-Bachet-Dameron-Chevalier;

- subject aged 8-15 years;

- subject presenting one or more ectopic maxillary permanent canines on a panoramic image at T0 (angulation $>10^{\circ}$ with respect to the median sagittal plane) $)^{4}$;

- subject has temporary canines. 


\section{Exclusion criteria}

The criteria for exclusion are:

- subject presenting a permanent canine with a visible anatomical defect;

- subject whose permanent canine is ankylosed;

- presence of anatomical obstacle on the eruption path of the permanent canine;

- Subject having maxillary endoalveolia;

- Subject has maxillary congestion;

- Subject having any maxillary agenesis;

- Subject wore orthodontic devices before or in conjunction with extractions of temporary canines.

\section{Judgment criteria}

The three main outcome measures are to evaluate the position of the maxillary permanent canine on a panoramic image:

- anteroposterior position of the permanent canine evaluated with respect to the long axis of the maxillary lateral incisor;

- angulation measured in relation to the median sagittal plane;

- vertical position evaluated in relation to the root of the maxillary central incisor.

The secondary judgment criteria assessed are the following:

- inflammation of the pericoronal sac of the permanent canine;

- rhizalysis of the temporary canine.

\section{Study method}

\section{Implementation of the protocol}

Once the clinical and radiological examinations of the patient have been carried out and the indication has been established, the extractions of maxillary temporary canines are recommended and must be performed rapidly by the oral surgeon.

The panoramic x-ray T0 is taken on the same day as the extractions are arranged. The primary and secondary judgment criteria are evaluated from this panoramic $x$-ray.

A radiological follow-up with a panoramic x-ray at 6-month intervals is scheduled over a maximum period of 18 months with evaluation of the primary judgment criteria (anteroposterior position, angulation, and vertical position) on each radiographic image.

In cases where the permanent maxillary canine is positioned on the arch before 18 months, radiological monitoring may be spaced further in the absence of other indications.

\section{Radiological evaluation}

Conventional radiological evaluation

The panoramic images are taken in the office of Dr. Gall-Bachet-DameronChevalier with the same device. The images are studied using OsiriX software ${ }^{\circledR}$. The primary judgment criteria are evaluated for each protocol step: $\mathrm{T}-0, \mathrm{~T}-6, \mathrm{~T}-12$, and $\mathrm{T}-18$, using the following method:

- Evaluation of the anteroposterior position of the maxillary permanent canine:

- plot of the major axis of the lateral incisor;

- plot of the tangent to the mesial edge of the lateral incisor;

- plot of the tangent to the distal edge of the lateral incisor;

- plot of the tangent to the distal edge of the temporary canine;

- plot of the major axis of the central incisor.

These five segments determine five sectors numbered from 1 to 5, from 
the most distal sector to the most mesial sector. Sector 1 is the most favorable for canine development, Sector 5 being the least favorable (Figure 1).

In this case, 23 is in Sector 3, so the score is 3.

- Measurement of the permanent maxillary canine angulation:

- plot of the median sagittal plane passing through crista galli and intermaxillary suture;

- plot of the long axis of the canine. The upper angle determined by these two lines is then measured. As the

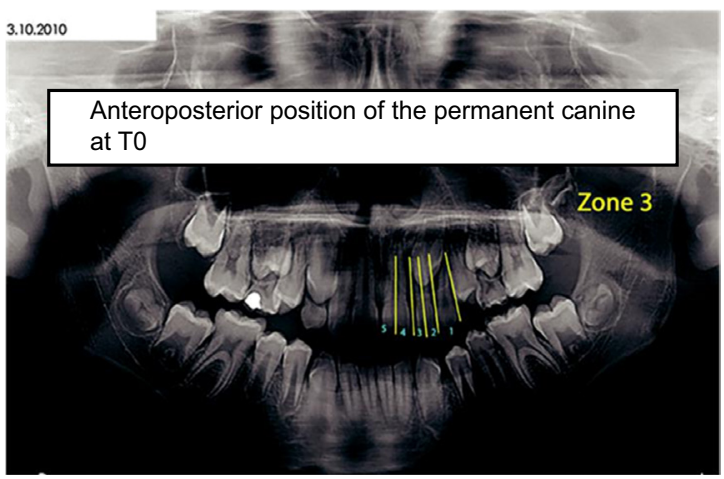

Figure 1

Evaluation of the anteroposterior position of the maxillary permanent canine.

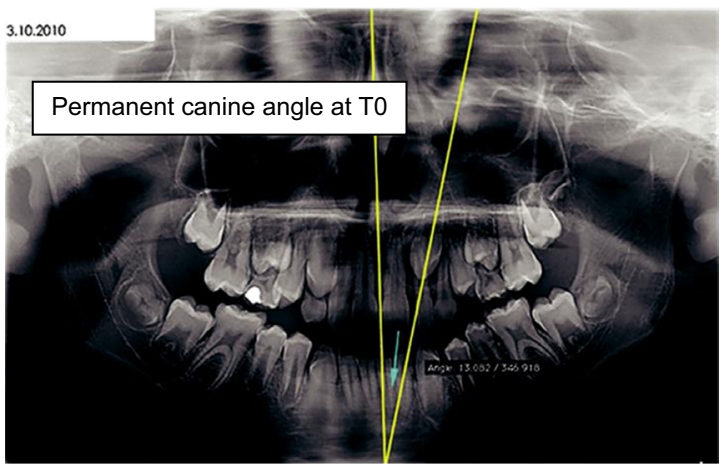

Figure 2

Evaluation of the angulation of the maxillary permanent canine with the median sagittal plane.

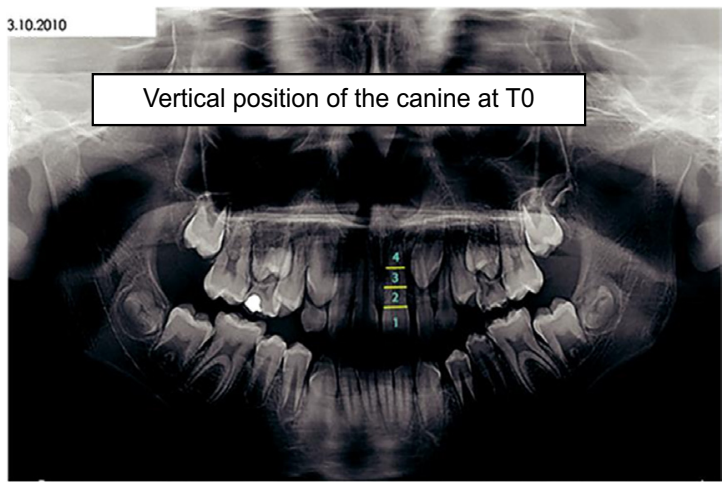

Figure 3

Evaluation of the vertical position of the permanent canine according to the central incisor.

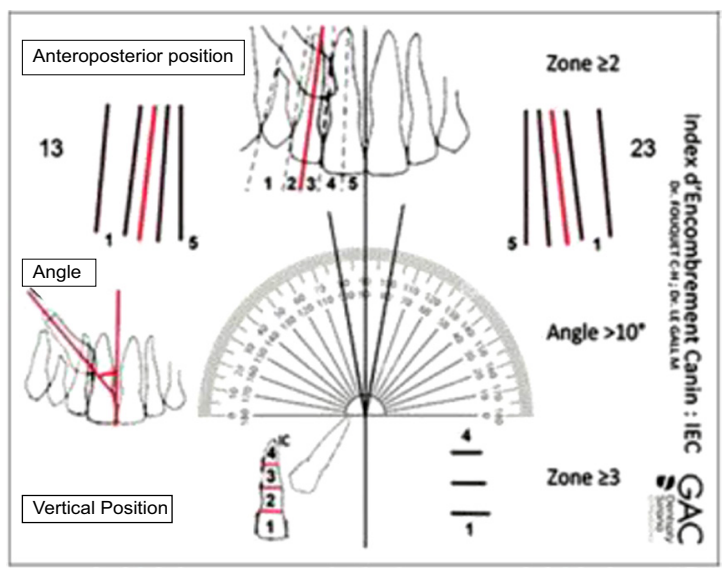

Figure 4

Canine Congestion Index (CCI) layer.

value of the angle increases, the position of the canine worsens (Fig. 2).

In this case, the 23 has an angulation of 13; hence, the score is 13.

- Evaluation of the vertical position of the maxillary permanent canine:

- Determination from the cementoenamel junction of the central incisor of three root segments of equivalent height.

These three segments determine four Sectors from 1 to 4 , from the most 


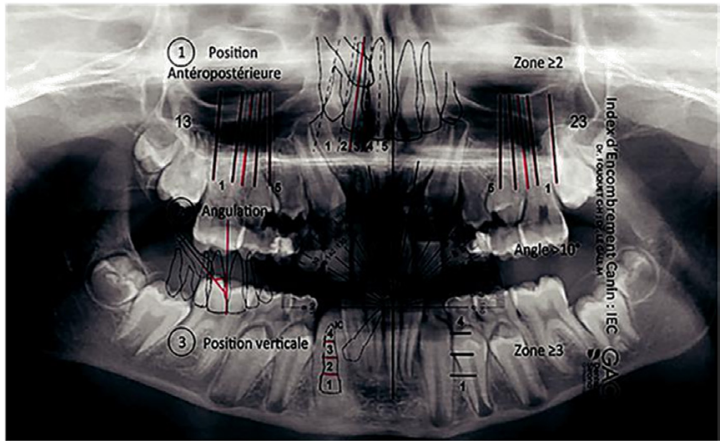

Figure 5

Placing the plot on the orthopantomogram.

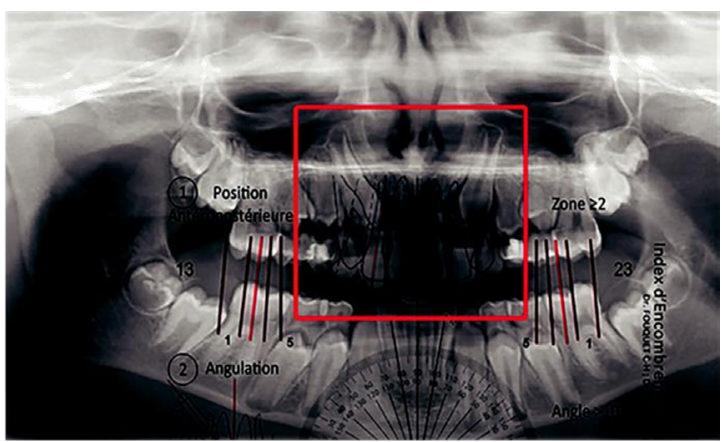

Figure 6

Superposition of incisor areas (radiograph and overlay) by zooming in or out.

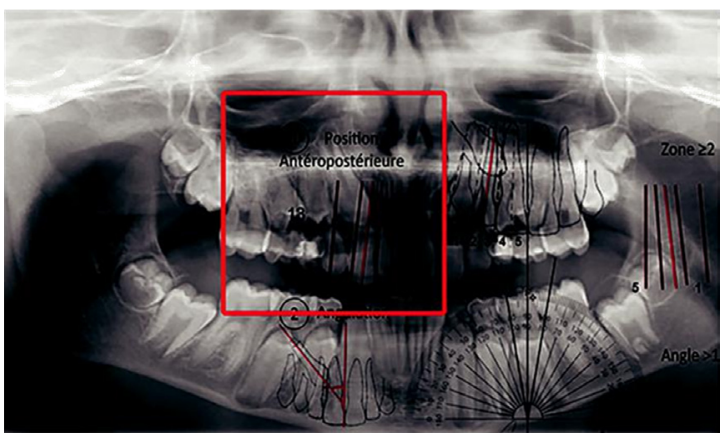

Figure 7

Evaluation of the anteroposterior position of the maxillary permanent canine.

occlusal sector (Sector 1 is coronal) to the most apical sector (Figure 3).

In this case, 23 is in Sector 3; so, the score is 3.
We have a total score of 19. The decision is made to extract the 63 .

The secondary judgment criteria were assessed on the TO image according to the following modalities:

- inflammation of the pericoronal sac: assessment of any increases in volume in the pericoronal sac;

- presence or absence of temporary canine rhizalysis.

It should be noted that the measurements were made on the 104 canines according to the same operating

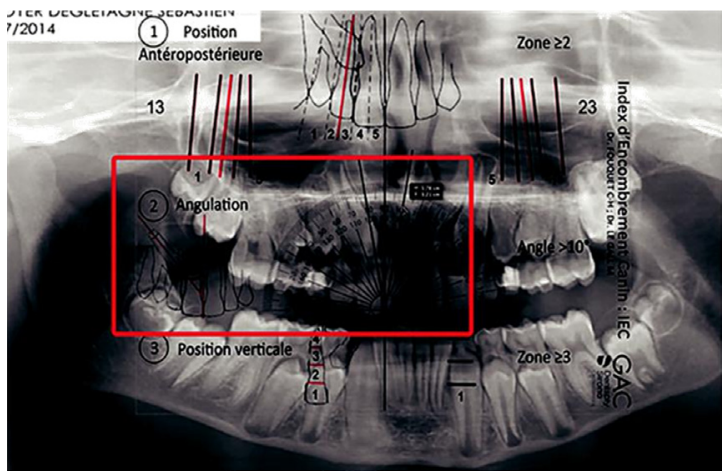

Figure 8

Evaluation of the angulation of the maxillary permanent canine with the median sagittal plane.

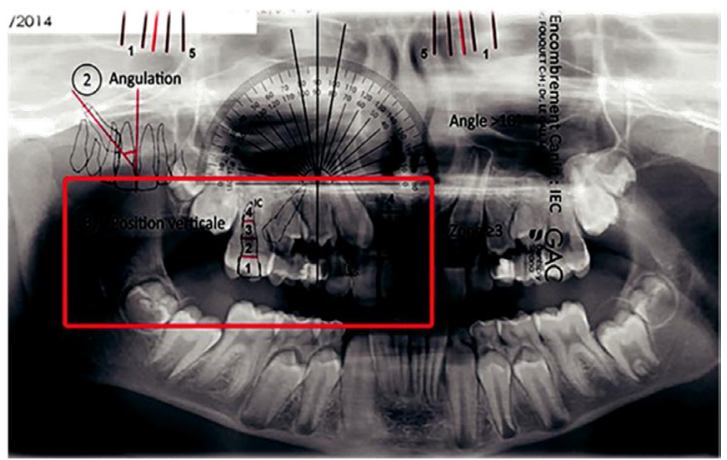

Figure 9

Evaluation of the vertical position of the permanent canine according to the central incisor. 


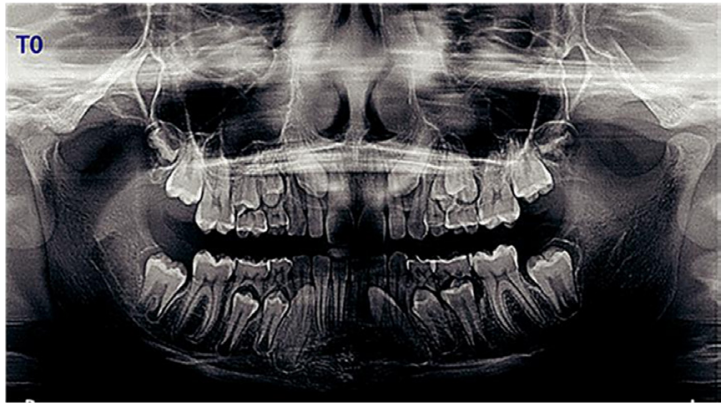

Figure 10

Patient orthopantomogram of 46 at TO.

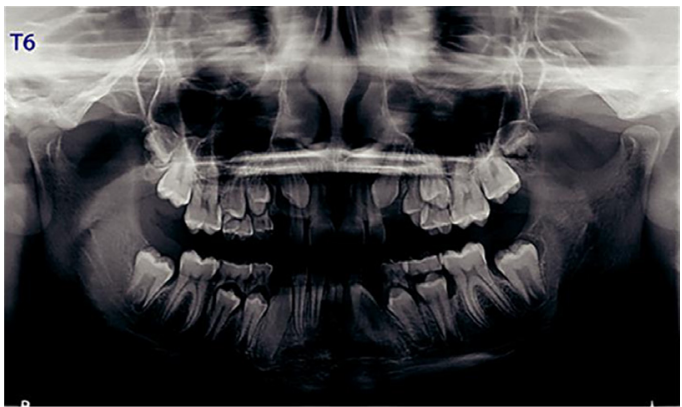

Figure 11

Patient orthopantomogram 46 to T6.

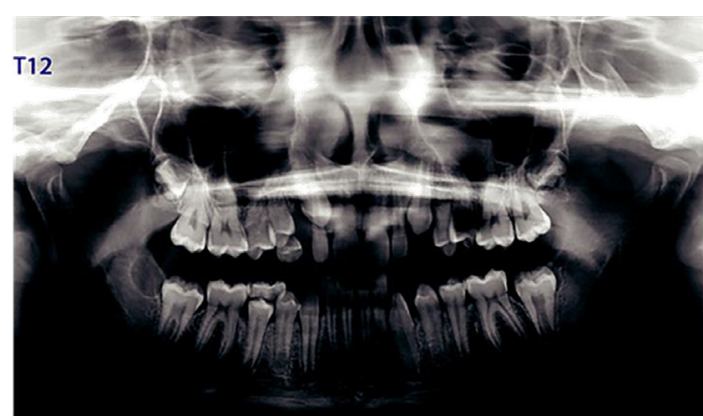

Figure 12

Patient orthopantomogram of 46 at T12.

procedure and by the same operator. The first 10 measures were performed twice by two different practitioners to respect intraindividual and interindividual variability.

Radiological assessment with the Canine Congestion Index $\left(\mathrm{CCl}^{\circledR}\right)$

Step 1: Scaling

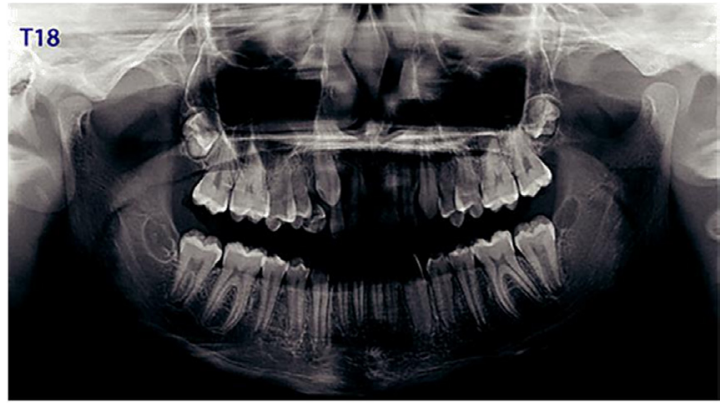

Figure 13

Patient orthopantomogram of 46 at T18.

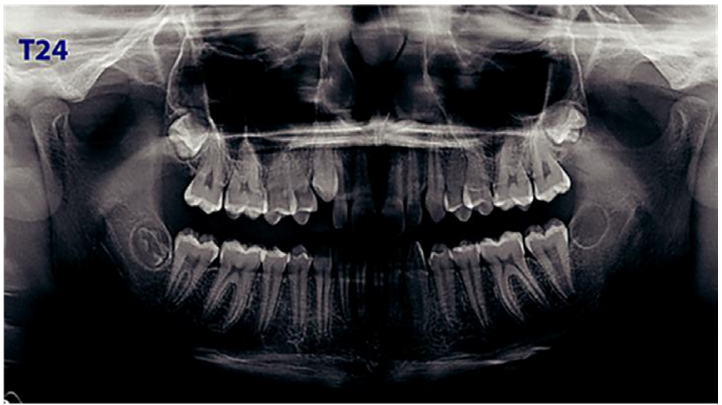

Figure 14

Patient orthopantomogram of 46 at T24.

Apply the $\mathrm{CCl}$ layer (Fig. 4) on your screen (Fig. 5).

Enlarge or decrease your panoramic radiograph so that the central area of $\mathrm{CCl}$ (Fig. 6) is superimposed on the incisal area.

Step 2: Evaluation of the anteroposterior position of the canine (Sector 1 of the layer) (Fig. 7)

Match the red line with the major axis of your lateral incisor. Note the area in which the tip of the canine is located. The sector determines the score.

Step 3: Evaluation of the canine angulation (Sector 2 of the layer) (Fig. 8)

Position the long axis of the protractor on or parallel to the PSM and directly measure the angle of the axis of the canine with this line. 
Step 4: Evaluation of the vertical position of the maxillary permanent canine (Sector 3 of the layer) (Fig. 9)

Position the lower line of the guide at the cementoenamel junction of the central incisor and note the area related to the tip of the canine.

Clinical case

Patient 46:

\section{RESULTS}

\section{Sample characterization}

Our sample consisted of 58 patients, 30 patients were female and 28 patients were male (Figure 15). This sample showed a slight female preponderance, which is consistent with the literature 7 .

The average age of our sample was 11 years, distributed as shown below (Figure 16).

We studied 104 maxillary canines, 49 were right maxillary canines (tooth 13) and 55 were left maxillary canines (tooth 23). In the literature regarding included canines, 23 is more often included than $13^{7}$ (Fig. 17).

In our study, the ectopic position of 13 and 23 is found in $78 \%$ cases (Fig. 18).

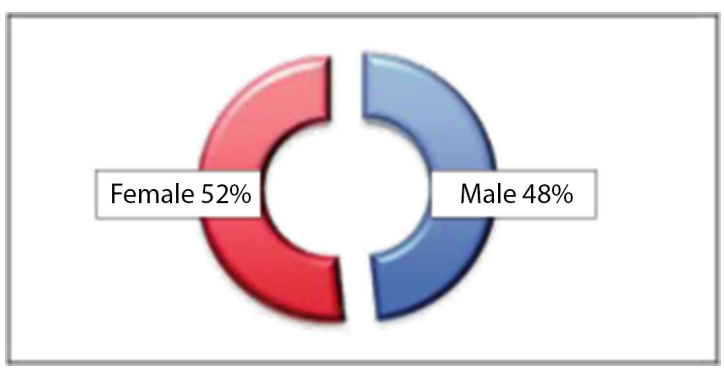

Figure 15

Distribution of the sample by gender.
- male;

- age 11 years;

- first consultation in October 2013 with extractions of $53 / 63$ and 73/83;

- radiological follow-up (orthopantomogram) every 6 months for 24 months in the absence of other orthodontic treatment (Figs. 10-14).

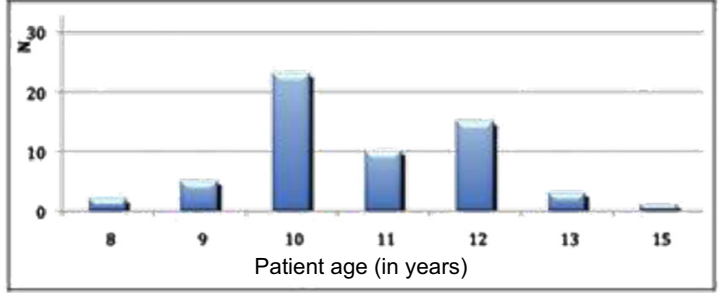

Figure 16

Distribution of the sample according to the patient's age at time of extraction.

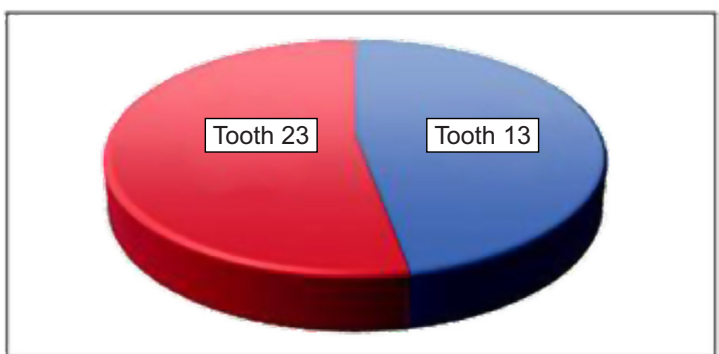

Figure 17

Distribution of the sample according to the ectopic tooth number.

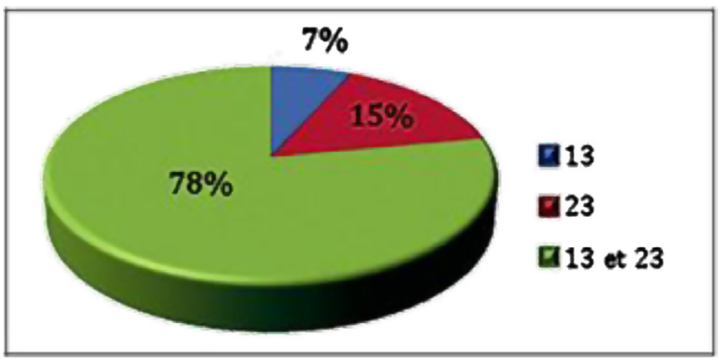

Figure 18

Uni- or bilateral distribution of canines in ectopic position. 
Evaluation of the anteroposterior position of the canines

Evaluation of the anteroposterior position of 13 and 23 over time showed an increase in the number of teeth present in Sector 1. The initial location of the canines was greater in Area 2 and it should be noted that no tooth belonged to Area 5. Over time, we observed a decrease in the number of

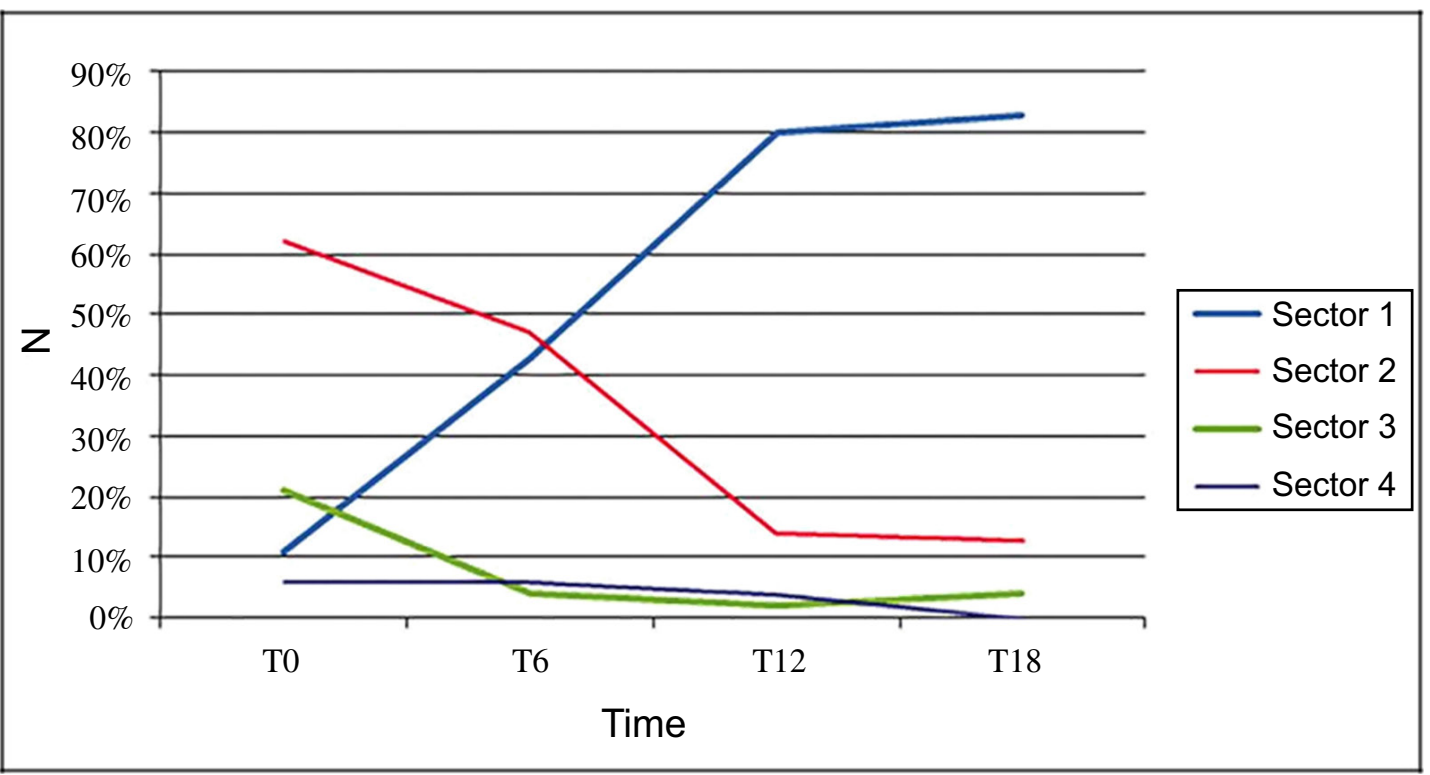

Figure 19

Evolution of canine percentage by anteroposterior Sector as a function of time.

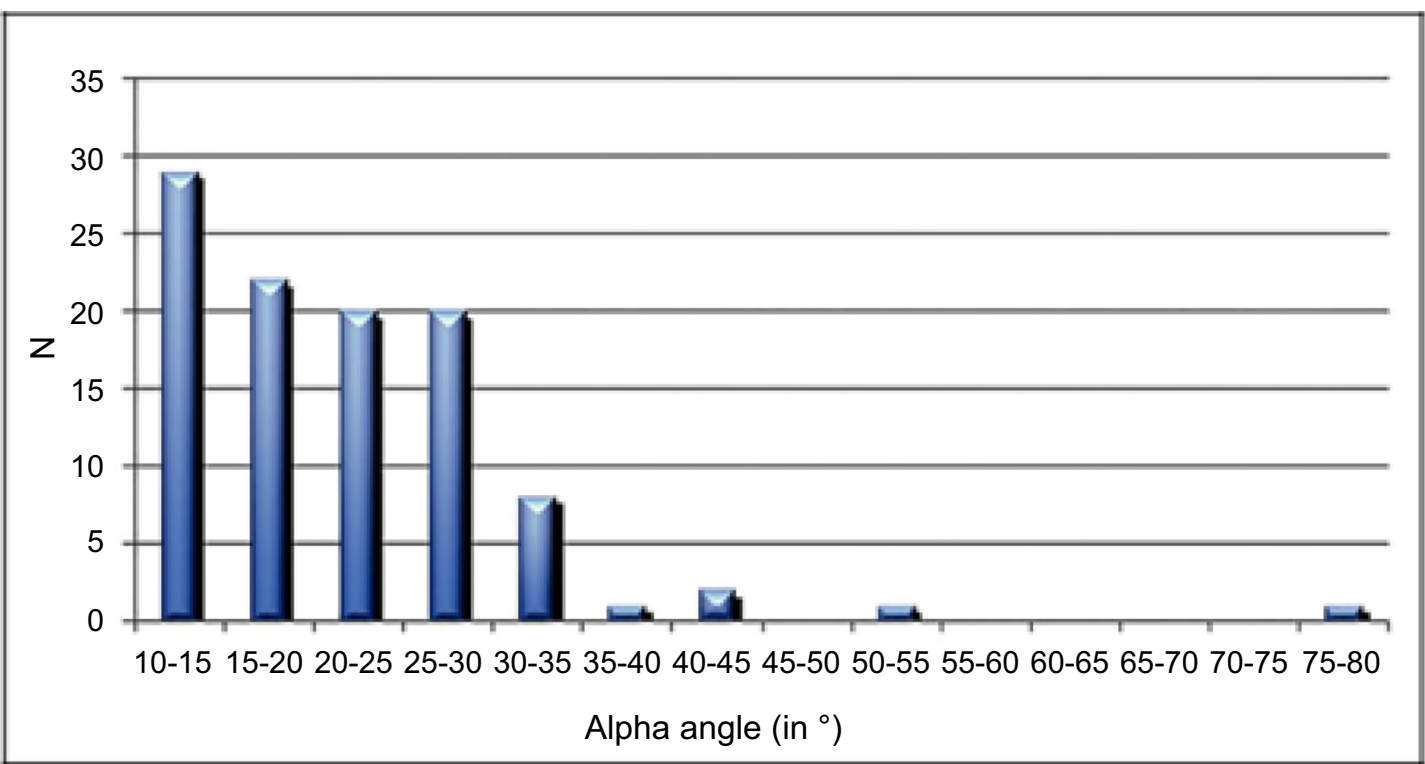

Figure 20

Initial distribution of alpha angles. 
teeth in Sectors 2, 3, and 4, with an increase in Sector 1 (with the exception of the Sector 2 proportion, which increased at T6 because of the decrease in the number of teeth in Sector 3 passing in Sector 2 at the first reassessment). In fact, we went from $11 \%$ (T0) to 83\% (T18) of canines in Sector 1 (Figure 19). Note that the improvement of the anteroposterior position stabilized between the periods T12 and T18 as shown by the curves (Figure 19).

\section{Evaluation of canine angulation}

The observation of the distribution of the initial values of the alpha angles showed a concentration of the inclination of the canines between $10^{\circ}$ and $30^{\circ}$ (average inclination $=21^{\circ}$ ) (Figure 20). We could note a decrease in the alpha angle during time with a very strong progression curve between T0 and T6 (Figure 21).

\section{Evaluation of the vertical position of the canines}

Evaluation of the vertical position of 13 and 23 over time showed an increase in the number of teeth present in Sector 1. Initial localizations of canines were more important in Sector 3 and it should be noted that no teeth belonged to Sector 5. Over time, we observed a decrease in the number of teeth in Sectors 2, 3, and 4 (with the exception of the Sector 2 proportion which increased at T6 because of the decrease in the number of teeth in Sector 3 passing in Sector 2 during the first re-evaluation), with a parallel increase in Sector 1. In fact, we go from $0 \%$ (T0) to $76 \%$ (T18) of canines in Sector 1 (Figure 22). Note that the improvement of the vertical position has stabilized between the T12 and T18 periods as shown in Figure 22.

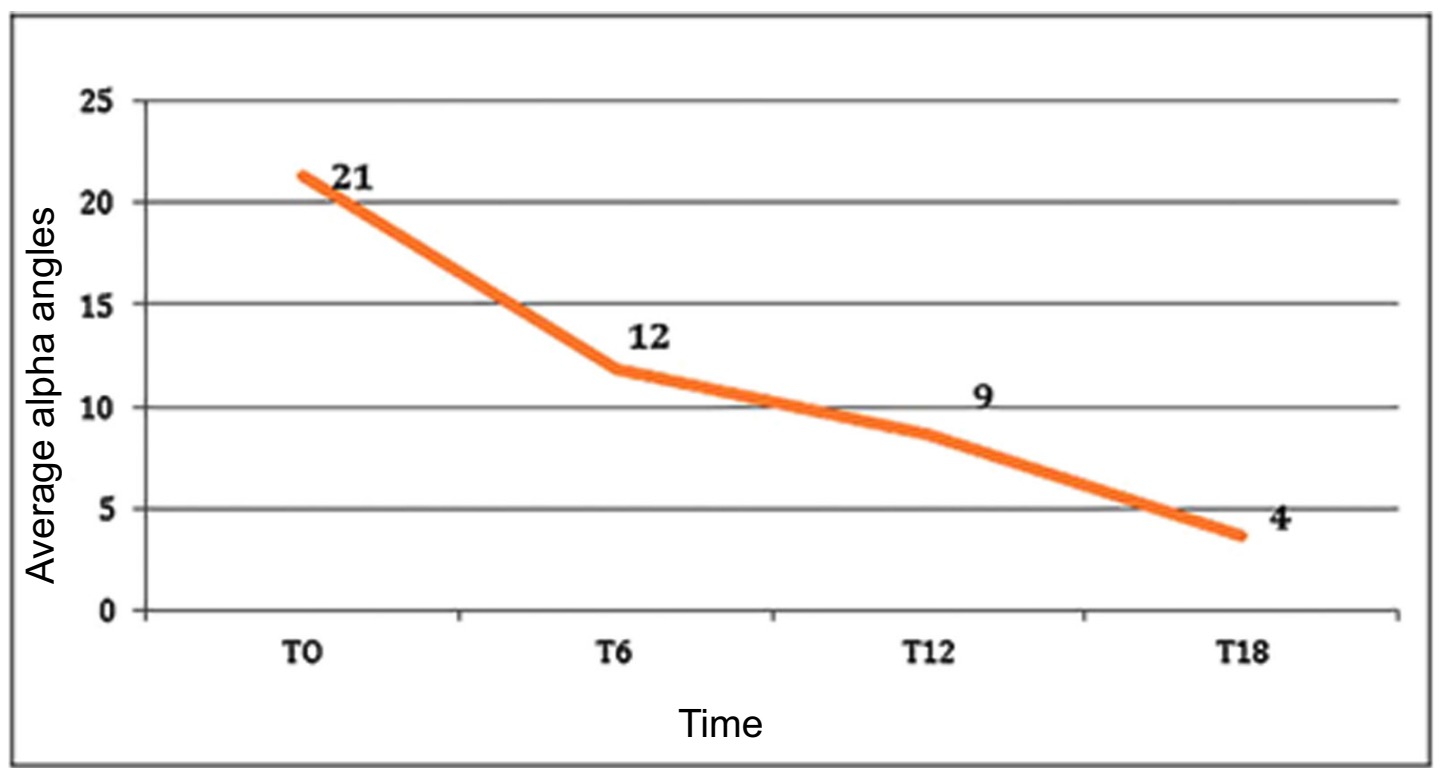

Figure 21

Average alpha angles of 13 and 23 as a function of time. 


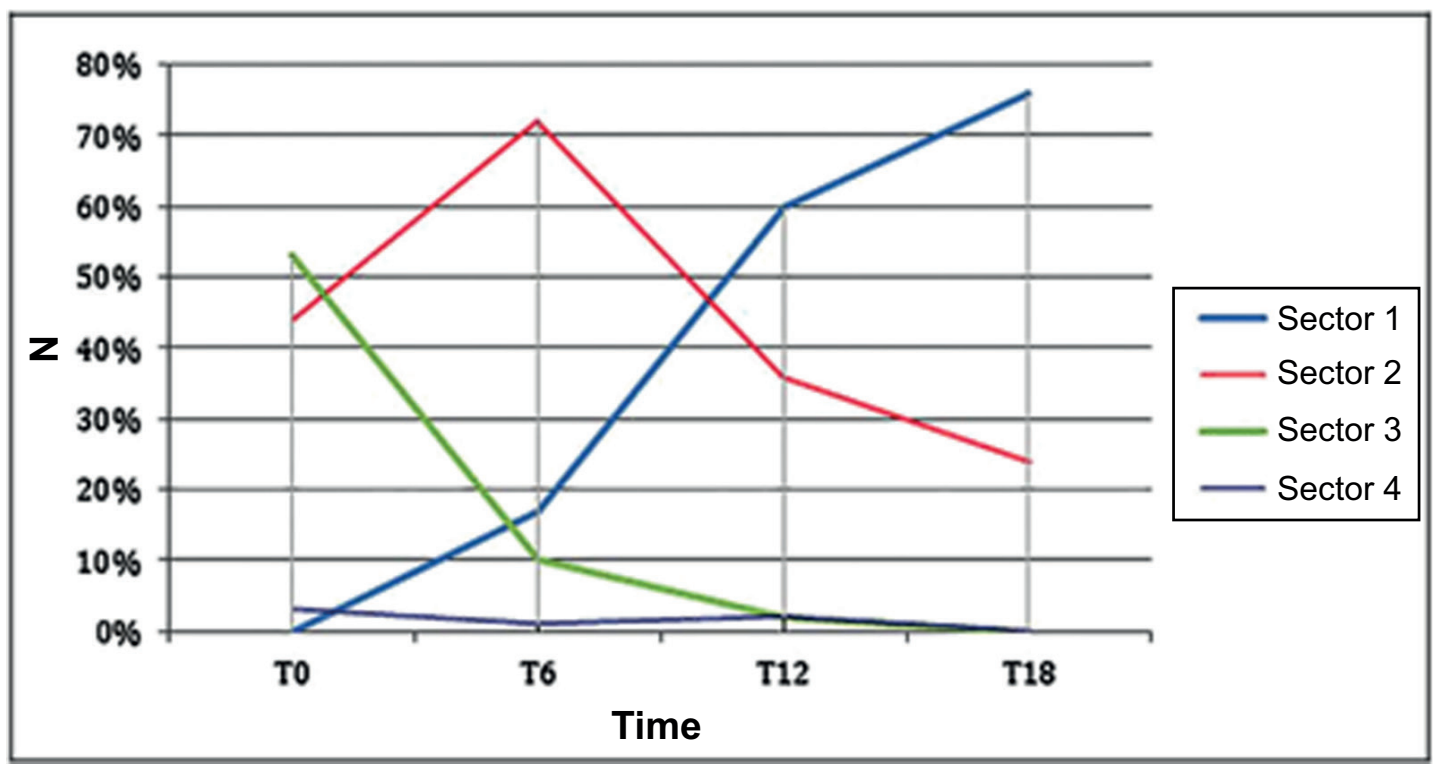

Figure 22

Evolution of canine percentage by vertical Sector as a function of time.

\section{Evaluation of the inflammation of the pericoronal sac}

During the initial observation (TO) of the 104 canines in the ectopic position, the size of the pericoronal sac was evaluated by the operator. When it was larger, it was considered inflammatory.

There was little difference between the number of canines with a noninflammatory and inflammatory pericoronary sac at T0 (Figure 23).

\section{Evaluation of the temporary canine rhizalysis}

The results of the initial observation (TO) of the temporary canine rhizalysis of the showed little difference between the number of temporary canines resorbed (at least partially) and not resorbed (Figure 24).

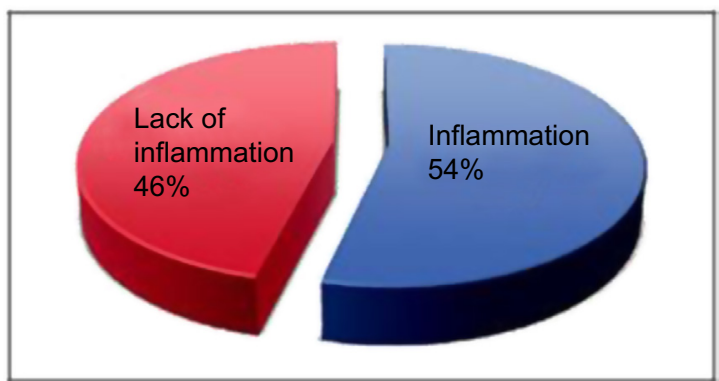

Figure 23

Percentage of initial inflammation of the pericoronal sac of 13 and 23 at TO.

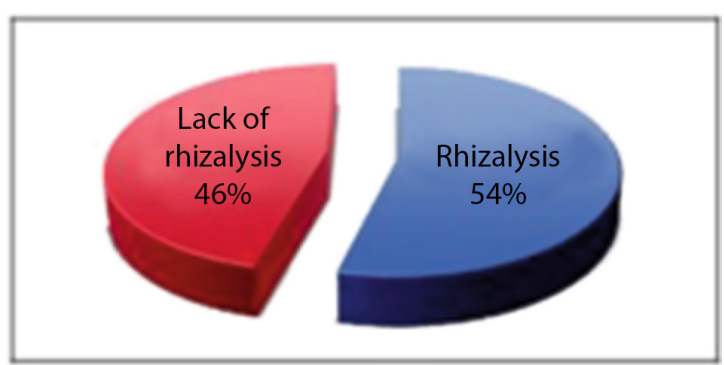

Figure 24

Percentage of rhizalysis of 53 and 63 at TO. 


\section{DISCUSSION}

The results of our study allowed us to recommend early extractions of temporary canines to avoid the impaction of permanent teeth.

In the literature, our main reference is the study by Ericson and $\mathrm{Kurol}^{2}$. Our study was conducted on a much larger sample (104 canines versus 46) without taking into account the initial position of the permanent canine (palatal, median, vestibular) and therefore not limited to only the palatal canines.

The study shows an improvement in canine position by only extracting temporary canines in $87 \%$ cases. The analysis of these cases reveals a similar average age (11 years) but there is an extreme outlier who is 15 years old. The failed group shows an initial anteroposterior position in the larger Sector 4. In the failed group, we also observe a greater initial angulation (TO) $\left(26^{\circ}\right.$ versus $\left.21^{\circ}\right)$, with a proportion of angulation $>30^{\circ}$. The initial vertical position in the failed group shows a higher percentage of canines in Sector 3 and 4. In this group, there is also a higher proportion of inflammation of the pericoronal sac and absence of rhizalysis of the temporary canine $(73 \%$ and $80 \%$ versus $54 \%$ and $46 \%$ ).

When there is no improvement, or it is not significant 12 months after the extractions, a therapeutic alternative should be considered (orthodontic or orthosurgical treatment). In fact, beyond 1 year, the improvement is much less significant.

So, in view of the results of this study, we can establish a $\mathrm{CCl}^{\circledR}$ for temporary canine extraction under the following conditions:
- patient aged 10-13 years meeting the inclusion criteria;

Canine with anteroposterior position $\geq$ Sector 2;

- canine with an angulation $>10^{\circ}$ from the median sagittal plane;

- a vertical position of the canine $\geq 3$.

The sum of these three measures gives you a score that is all the more important as the risk of impaction is high.

If the score is $\geq 15$, the extraction of the temporary canine is indicated to improve the spontaneous spatial repositioning of the permanent canine.

The following signs can also lead to interceptive treatment:

- an increase in the volume of the pericoronal sac and/or;

- The absence of rhizalysis of the temporary canine not consistent with the dental age of the patient.

Radiological monitoring should be done every 6 months with the most reproducible panoramic images possible (same device, same operator).

Avoiding the impaction of canines through the simple analysis of orthopantomogram images is essential for both the patient and the practitioner. Before the age of 10 years, a spontaneous improvement in the position of the permanent canine can be observed without extraction of the temporary tooth. However, if the position of the permanent canine is unfavorable, the extraction can be performed to obtain at least an improvement of the initial situation. The presence of an included canine increases the duration of treatment by at least 1 year (on average 12-14 months) ${ }^{3}$. Even if temporary canine extraction alone 
does not prevent impaction, the improvement in the alpha angle obtained in a very large majority of cases significantly decrease treatment time when it is $<25^{\circ 3}$. The significant risk of resorptions of the adjacent teeth to the included teeth is now clearly proven and should encourage us to prevent impactions. In addition, orthosurgical setup failures of the included canines are not uncommon, periodontal damage can be disastrous, and the discomfort suffered by the patient is significant ${ }^{5,6}$.

\section{CONCLUSION}

Our study has shown that the extraction of temporary canines at an early age can rapidly and significantly improve the position of ectopic maxillary canines and prevent impactions.

The detection of this malposition must be done when patients are young to maximize our chances of success using this technique.

Our results have allowed us to predict a very high success rate, but interindividual variations exist, which will sometimes result in failures in the absence of any initial signs.

It is essential that a Qualified ODF Specialist is consulted before the age of 10 years for an orthopantomogram
In order to better evaluate the indications of this treatment, it would be necessary to carry out a comparative study with an untreated control group. However, for ethical reasons, it seems difficult to conduct such a study because of the patient's loss of chance to correct future problems. A prospective study on impactions according to the clinical indications previously defined by this study to avoid recruitment bias is currently being initiated by Dr. Laura David under the supervision of Dr. Michel Le Gall. to ensure the early detection of clinically silent problems. The radiological assessment should be performed at 6-month intervals in a reproducible manner to study the position of the canine. The lack of significant improvement over a period of 12 months post-extraction requires a re-evaluation of therapeutic alternatives.

Before making any decision to prematurely extract temporary canines, the clinical case must be evaluated in its entirety in order to verify that it corresponds to the indications of this therapy.

Conflict of interest: The authors declare that they have no conflict of interest.

\section{BIBLIOGRAPHY}

1. Delsol L, Orti V, Chuvin M, Canal P. Canines and maxillary incisives included: diagnosis and therapy. EMC - orthopedics private 2008. 
2. Ericson S, Kurol J. Early treatment of palatally erupting maxillary canines by extraction of the primary canines. Eur J Orthod 1988;10(4):283-95.

3. Giuly AB. Prospective study on 127 maxillary included canines: duration of treatment and characterization of the population. Orthodontics Bioprogressive 2015;23:19-27.

4. Korbendau JM, Patti A. Orthodontic and surgical treatment of the included teeth. Quintessence International, 2005.

5. Oliver RG, Hardy P. Practical and theoretical aspects of a method of orthodontic traction to unerupted teeth illustrated by three cases. BR J Orthod 1986;13(4):229-236.

6. Ackerman JL, Proffit WR. Soft tissue limitations in orthodontics. Angle Orthod 1997; 67:327-336.

7. Syryn'ska M, Budzyn'ska A. The incidence of uni- and bilateral impacted maxillary canines and their position in the human arch. Ann Acad Med Stetin 2008;54(2):132-137. 